\title{
Research on Design of Fog Computing Optimization Model for Medical Big Data
}

\author{
Baoling QIN ${ }^{1}$ \\ College of Electronic Information Engineering, Foshan University, Foshan, China
}

\begin{abstract}
Targeted at the current issues of communication delay, data congestion, and data redundancy in cloud computing for medical big data, a fog computing optimization model is designed, namely an intelligent front-end architecture of fog computing. It uses the network structure characteristics of fog computing and "decentralized and local" mind-sets to tackle the current medical IoT network's narrow bandwidth, information congestion, heavy computing burden on cloud services, insufficient storage space, and poor data security and confidentiality. The model is composed of fog computing, deep learning, and big data technology. By full use of the advantages of WiFi and user mobile devices in the medical area, it can optimize the internal technology of the model, with the help of classification methods based on big data mining and deep learning algorithms based on artificial intelligence, and automatically process case diagnosis, multi-source heterogeneous data mining, and medical records. It will also improve the accuracy of medical diagnosis and the efficiency of multi-source heterogeneous data processing while reducing network delay and power consumption, ensuring patient data privacy and safety, reducing data redundancy, and reducing cloud overload. The response speed and network bandwidth of the system have been greatly optimized in the process, which improves the quality of medical information service.
\end{abstract}

Keywords. Fog computing, optimization model, medical system, big data

\section{Introduction}

With the continuous advancement of the Internet of Things in all areas, an intelligent society is gradually taking shape in front of our eyes. At the same time, the demand for data processing closely related to intelligence has become higher and higher, especially in some specific fields such as medical information. With the continuous upgrading of medical intelligence, the data generated is becoming larger and larger. The traditional big data processing through cloud computing can no longer meet the needs of the current speed of medical big data processing, especially for multiple sources as the data structure is very complex. Besides, there are problems with computing and storage. The cloud-computing-oriented medical system processes information from different edge devices at the same time. The heavy burden, the network delays, and so on lead to the poor performance of cloud computing, increased network delays, and even system failures. Aiming at solving these shortcomings of medical big data in the Internet of Things, a fog computing optimization model is designed. The model extends computing from the cloud to edge devices and uses the distribution characteristics of

1 Corresponding Author, Baoling QIN, College of Electronic Information Engineering, Foshan University, Foshan, China; Email: 2978879663@qq.com. 
fog computing to reduce cloud computing and storage overhead. Numerous simple calculations are handed over to fog computing, thus reducing unnecessary data processing on the network. The cloud only takes care of scheduling and comprehensive computing work, thereby improving the response speed of the medical system and network bandwidth. It can reduce network latency and power consumption, relieve cloud computing and storage pressure, ensure network information security, make full use of the advantages of fog computing, deep learning algorithms, and big data technology, optimize system models, and promote the intelligent construction of medical big data systems. The significance is pragmatic and far-reaching.

\section{Fog Computing Network System and Its Architecture}

Fog computing is an extension and the supplement of cloud computing. It is characterized by decentralized localization and a distributed computing model [1], which distributes computing, storage, network, control, and decision-making functions close to users. It occupies an important position in the Internet of Things, with a computing mode of modular distributed computing structure.

\subsection{Concepts and Characteristics of Fog Computing}

Fog computing is a concept that was first formally proposed by Cisco in 2011. It attempts to fix the issues of network delay, network bandwidth and reliability caused by the increasing deployment and huge data of the Internet of Things. It is an extension of the concept of cloud computing, a new generation of distributed computing mode that places some data in "local" devices at the edge of the network for processing. It utilizes a large number of network edges that have weak computing and storage capacities at the edge of the network to store and calculate some data, and then uploads useful data to the cloud. It features "decentralization and localization", which can transmit data with extremely low latency. It consists of a large number of scattered weak network edges. At the same time, it also has excellent mobility, allowing communication devices and mobile devices to communicate directly with each other, which is an ideal model architecture for the Internet of Things.

\subsection{Fog Computing Network Architecture Based on the Internet of Things}

Fog computing is an emerging distributed computing model for big data and the Internet of Things. It is not composed of powerful servers, but of huge edge devices with inferior performance and wider distribution located outside large data centers. Its characteristic is mobility and distribution at a larger range, adapting to more and more intelligent devices such as medical terminal equipment that do not require a large amount of calculation. The purpose is to improve the atomization of computing efficiency and reduce the problems of data transmission to the cloud for localized processing, analysis and storage. Fog computing provides the Internet of Things system with a three-low network data service quality [2], which means low latency, low power consumption, and low traffic. It adopts decentralized, localized and distributed cluster computing strategies to make the Internet of Things system more suitable for the calculation, analysis and standardization of multi-source heterogeneous data, and 
enhances the computing capacity and storage of the Internet of Things system. In short, the network architecture of fog computing in the Internet of Things system can be divided into three layers [3], the user layer, the fog layer and the cloud layer, as shown in figure 1.

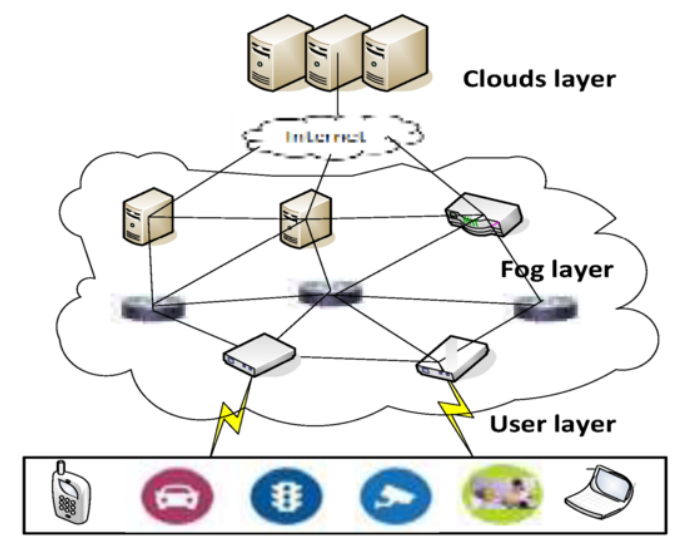

Figure 1. Fog computing network architecture based on the Internet of Things.

\section{(1) User layer}

The user layer is the bottom layer in the fog computing network architecture model. It is composed of numerous smart terminals and massive data generated by them. Through short-distance communication such as WiFi and Bluetooth, fog computing users can connect smart terminals with high-level fog nodes to enjoy the services provided by the fog computing network [4].

The terminal equipment in the medical system includes electronic sphygmomanometer, electronic thermal probe, CTS equipment, B-ultrasound equipment, etc., whose main goal is to collect the required data information through different terminal equipment with different technologies, send the information to the fog node, and then gather information to the fog layer. In addition to collecting information, the sensor layer also includes the networking of sensor devices that can improve the survivability and flexibility of the network, and enhance the ability to interfere with data during transmission. These technologies mainly concern sensor technology, RFID technology, scan code technology, ZigBee and Bluetooth technology [5].

\section{(2) Fog layer}

The fog layer is located in the middle layer of the Internet of Things system and deployed on the scattered fog servers. It acts as a bridge between smart terminals and the cloud. Each fog server is a highly virtualized computing system, similar to a lightweight cloud server, with a variety of wireless data storage, processing, and communication devices for intelligent processing of data loaded to peripheral devices, including peripheral devices and regional processing levels.

(a) The edge computing layer is the level where the fog node exists. The fog node is a random edge terminal device with certain computing capabilities, such as a PC. The sensor layer forwards the collected data to the fog node, processes the information through the fog node, and returns the completed result to the corresponding terminal device and transmits the unfinished task to the fog server. Numerous fog nodes form 
the edge computing layer, and all fog nodes are interconnected. As edge terminal devices with certain computing capabilities can be selected as fog nodes, the edge terminal devices that become fog nodes can realize processing localization, which greatly improves the accuracy and timeliness of medical data information, free from the delay and communication overhead caused by the distance.

(b) The regional computing layer is the level where the fog server exists. The fog server is an edge terminal device node with stronger computing and storage capabilities, such as high-performance routers, high-performance network communication equipment, etc. The fog server is random. The nodes are all connected to one other, forming a regional coverage with many fog servers and fog nodes in an area. Since all fog nodes are dynamic, the equipment fault tolerance rate of the fog computing layer is very high. The fog server conducts the communication connection with the cloud, and sends the computing tasks and completed task information that the fog computing layer cannot complete to the cloud and receives the information returned by the cloud and sends it back to the corresponding terminal device. Since a lot of medical information is actually not considerable when processed separately, much of it can be completed in the fog computing layer, so that the amount of data transmitted to the cloud is greatly reduced, and the computing and storage pressure of the cloud server is greatly relieved. In addition to its own computing function, the fog server has the function of realizing distributed computing, dynamically assigning computing tasks to vacant or capable computing fog servers and fog nodes. Thanks to the fact that all nodes in the fog computing layer in this model are dynamic, it makes distributed computing easier and more efficient to implement. The entire regional computing layer is the core of the fog computing network architecture.

\section{(3) Clouds layer}

This layer is the highest layer in the fog computing network architecture. In the system, the ability of fog equipment to calculate and process data is very limited. When there are tasks that require more computing resources, the user data to be processed will be uploaded from the fog layer to the cloud layer through a high-speed network. Powerful cloud computing functions are used to provide richer resources and applications.

The cloud server is a server cluster that relies on cloud computing and has extremely high performance. It has powerful computing and storage capabilities. It can complete computing tasks that cannot be completed by the fog computing layer and return the results. It can store data results obtained through collection in the cloud, and the cloud computing layer can also use cloud computing and data mining. The medical information obtained is analysed, and the results are used to help doctors diagnose the patient's condition, formulate corresponding medical plans and medical research, etc., which greatly raises the work efficiency of medical staff and the quality of medical services [6]. Since cloud servers are usually far away from medical terminal equipment, there is a delay in network data and huge communication consumption. And fog computing can well alleviate this problem. The powerful computing and storage capabilities of the cloud layer are an important part of the IoT system.

\section{Fog Computing Optimization Model Design for Medical Big Data}

With the widespread application of medical big data, the complexity of big data processing becomes more and more prominent in the current cloud computing model. 
For example, the medical system needs to process a large number of heterogeneous data from multiple sources, network delays, and central computing overload. Thus, it is necessary to design a fog computing optimization model through big data technology and fog computing characteristics, and adding deep learning methods to internal algorithms.

\subsection{Problem Analysis}

For the current medical system, the data sources are diversified, the network is real-time, and the intelligence requirements are high. The cloud server and the underlying terminal equipment are usually far from each other. Although the cloud computing and storage capabilities are strong, the distance band cannot be avoided. Incoming data delay and huge communication overhead lower the accuracy and timeliness of data information; at the same time, due to the huge number of terminal devices in the medical field, when they need to access the cloud server, it causes the cloud server to be overloaded and the network to fail. The doctor's efficiency cannot be guaranteed [6].

This computing mode relies on fog computing as the connection point to connect cloud servers to underlying edge devices, and uses distributed computing to exploit the underlying edge device resources, improve the instantaneity, accuracy and effectiveness of data, and ease the huge pressure of data processing and data storage of the cloud server. In this model, the bottom edge device is not directly connected to the cloud server, but uses the fog computing layer as a transit station to transmit data to the cloud server and the bottom edge device, in a (bottom edge device) $\leftrightarrow$ (fog computing layer) $) \leftrightarrow$ (cloud server) data transmission mode. The fog computing layer has its own server, which can perform a certain amount of data processing and data caching. Although the performance of the server is not high, it can be the underlying edge device with the benefits of localization, low latency, low price, and highly cost-effective. It can realize real-time local data processing, add deep learning algorithms to the fog layer server, and improve multi-source heterogeneous data processing capabilities and data standardized output capabilities. Furthermore, patient privacy data will not be uploaded to the cloud server for processing, and can be processed on the fog server, which effectively guarantees the security of private data.

\subsection{Design of Fog Computing Optimization Model for Medical Big Data}

The fog server is composed of hardware and software systems, where the hardware system includes: processor module, memory module, power management module and communication interface module; software system includes: operating system, Web system, database system and programming language system, as shown in figure 2 . The key technologies of the fog server in the medical system are WiFi Network Access and Information Query and Search. The former is the basis for the server to provide services for various mobile applications. For large-scale Grade III Level A hospitals, it needs to access multiple mobile service points at the same time, and requires high network bandwidth. Then, the wireless spectrum network access mechanism is designed here to improve the autonomous access method of mobile users. The mobile user equipment adopts active scanning and passive monitoring methods to detect the local WiFi network to ensure the smoothness of the network. The latter is that the server uses the local information fusion query mechanism, that is, users can transact 
business at any window, thereby reducing patient queuing time and improving service quality.

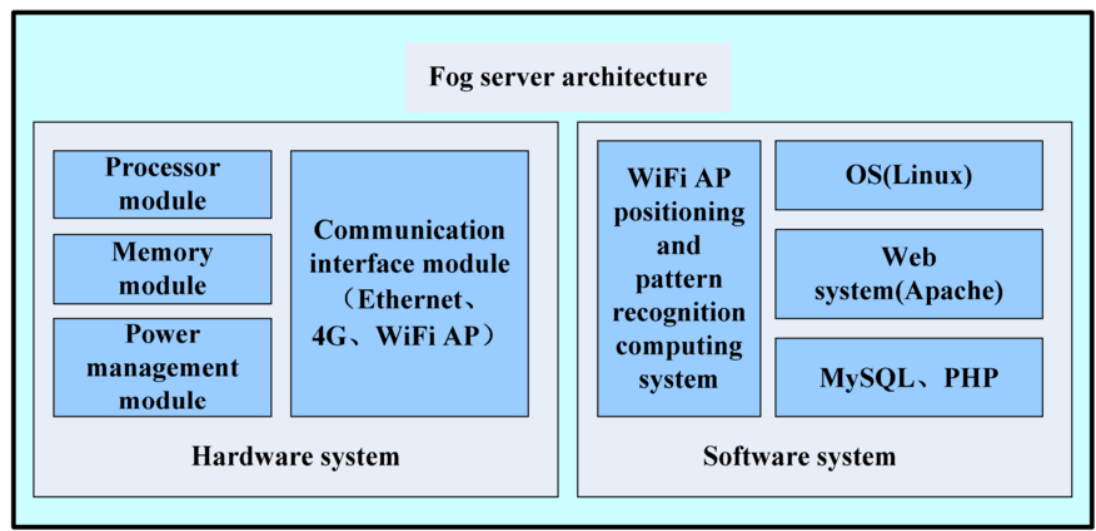

Figure 2. Fog server architecture.

In the medical system, the processing and transmission of large amounts of data generated by medical terminal equipment face huge challenges, such as multi-source heterogeneous data processing and network transmission delay. However, with the introduction of the concept of fog computing and the widespread application of deep learning of $\mathrm{AI}$ in recent years, medical big data processing has new research directions. In view of the complexity and particularity of the data processing of medical big data, the modular design method is adopted for the function design of the fog computing model, which effectively guarantees the function stability of the fog computing layer, and also effectively improves the scalability of this layer. The functions of the layer include four modules including heterogeneous gateway, data processing, resource management and classification management, as shown in figure 3.

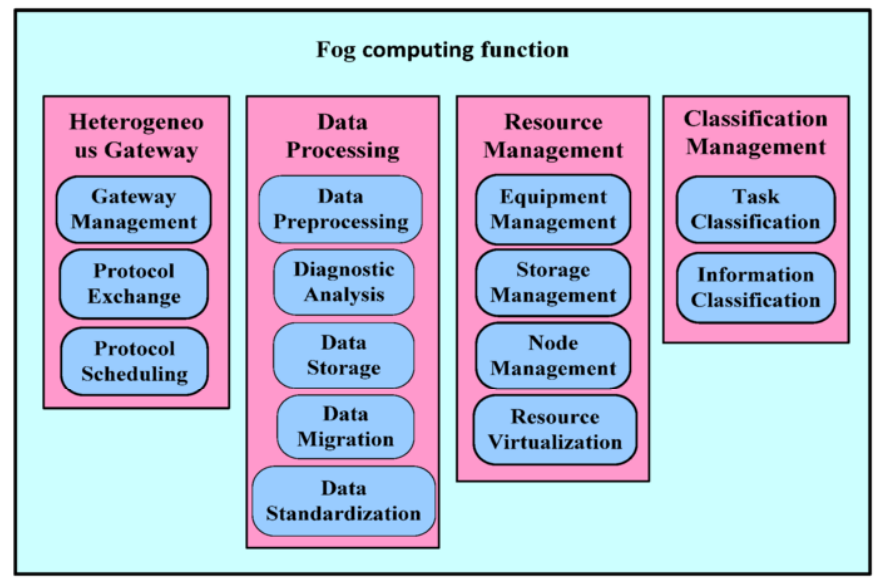

Figure 3. The fog computing function model in the medical system.

The medical system has the characteristics of human-machine-object interaction [7], where (1) Cloud computing and the Internet are infrastructure; (2) Internet of 
Things is an interactive method; (3) AI is an application mode; (4) Big data is an information processing technology. The integration of fog computing and big data technology is the core part of the medical system. Since the processing and storage of multi-source heterogeneous data in the medical system requires ultra-low latency and real-time processing, it is necessary to design a distributed intelligent "fog computing + deep learning" model, which mainly includes fog computing, big data technology and deep learning algorithm, as shown in figure 4.

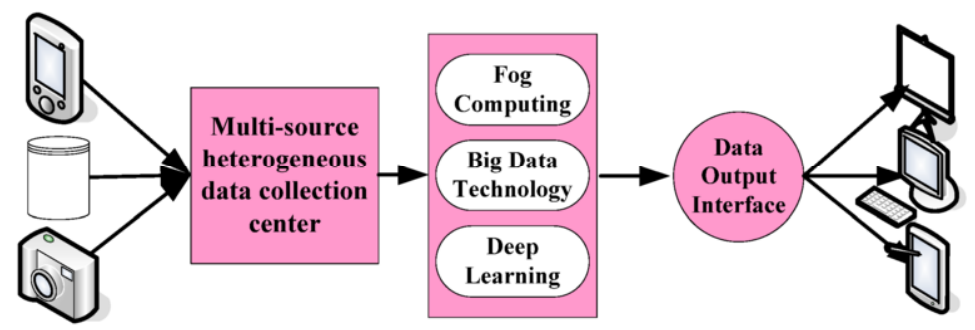

Figure 4. Multi-source heterogeneous data processing model.

Multi-source heterogeneous data has the characteristics of multi-source, heterogeneity and complexity. Big data technology is used to preprocess, structure and standardize these data, and fog computing and deep learning algorithm are combined to solve the problems in medical system, such as delay, storage, calculation, analysis and sample selection. In the fog server, the classification technology based on big data mining is used to diagnose the disease, and the deep learning algorithm based on AI is used to infer, analyze and predict the development of the disease. Diagnosis results are displayed in data visualization, web page mode, and other ways to facilitate doctors to understand the results and provide doctors with correct decision-making evidence. In this way, the medical big data platform can better serve medical institutions.

In general, in the traditional cloud service model, user information needs to be actively sent by mobile devices to the cloud server for identification and calculation, and when users need information, they must be obtained from the cloud server. Both processes are time-consuming on the network and increase network bandwidth. The pressure consumes cloud server computing and storage resources. In the application, since data generation and consumption are completed in the same area, using the characteristics of the fog server and the integration mechanism of local information and local computing resources, most of the data is processed directly on the fog server. This avoids the transmission of a large amount of raw data to the cloud server, reduces the transmission of a large amount of data back and forth on the network, and reduces their calculation and storage on the cloud server. Give full play to the advantages of fog computing in localized data processing, and introduce artificial intelligence deep learning algorithm technology into disease diagnosis to perform automatic judgment and processing, thereby improving the accuracy of medical diagnosis results and the effect of medical big data processing, and ensuring the quality of medical services.

\section{Case Analysis}

At present, the research of the "fog computing + deep learning" model in the medical 
big data system is in its infancy, which can be the core computing of the smart medical system, and it will take time to fully promote the application.

\subsection{Application and Significance}

With the rapid development of the application of information technology in the medical field, the data of the smart medical system has exploded. The ever-increasing amount of data and patients makes the workload of doctor's increase day by day, resulting in tension between doctors and patients. Although the application of big data technology has alleviated some medical problems, the problems are far from being solved completely. How to improve the work efficiency of doctors has become an urgent problem [8]. The purpose of designing the fog computing model is to integrate fog computing and big data in the medical field. It utilizes the edge localization characteristics of fog computing to reduce the pressure of computing and storage of big data in the cloud, improve the use efficiency and data accuracy of edge devices, and reduce the network delay and data loss of data transmission. Its application can achieve more accurate data and shorter processing time, so that edge devices can better assist doctors in their work and improve work efficiency.

\subsection{Requirements of the Medical System for the Fog Computing Optimization Model}

The medical system is a large-scale comprehensive system, which includes many subsystems, such as HIS system, RIS system, database system, etc. [9]. The amount of data in each subsystem is very large and complex. The medical system has strict requirements for data accuracy, timeliness, and reliability, and requires better collaboration between various subsystems, so the medical big data system has the following requirements for the fog computing model:

(1) Extremely low transmission delay and jitter. The edge computing of the model can achieve extremely low round-trip delay of data transmission or realize localized processing.

(2) Strong integrity. The three layers (cloud layer, fog layer, and sensor layer) are coordinated seamlessly, and data can be freely transmitted and shared in the model.

(3) High reliability. The model supports edge processing to make the data more accurate.

(4) Lower resource consumption. Compared with the pure cloud computing model, the fog computing model consumes lower resources and makes full use of the resources of edge devices.

(5) Strong compatibility, allowing different devices and data transmission technologies to coexist in the model.

(6) Good scalability. The model allows all fog nodes to join and exit at any time.

(7) Wide coverage. There are a large number of fog nodes in the model, whose geographical distribution is very wide.

(8) High fault tolerance. The model requires the fog nodes to form a network by themselves to complete the topology, achieve dynamic task allocation and dynamic data transmission, and have a high fault tolerance rate for edge devices.

\subsection{Advantages of Fog Computing Optimization Model}

With the rapid development of economy, technology and society, people pay more 
attention to health and medical security issues, and the demand for smart medical care becomes more urgent. Medical institutions have begun to use big data technology and AI medical diagnosis systems in large quantities. However, due to the characteristics of big data technology, these systems will have some limitations in application. Therefore, a medical big data system based on fog computing has been proposed, whose advantages are as follows:

(1) Very low time delay and relatively low jitter

The fog computing layer is a layer with data processing capabilities and data storage capabilities between the cloud computing layer and the terminal device layer. The fog node is lower in the network topology and closer to the edge of the network. Fog computing works at the edge of the local network. Compared with cloud computing, it suffers less interference and lower jitter [10]. For data access services, fog computing does not require data access to the cloud. On the contrary, it is directly accessed on local devices and mobile devices, which greatly shortens the transmission distance. At the same time, it accesses data in a stable and extremely low-latency manner, which greatly improves the efficiency of data transmission. It can be seen that the fog computing technology is very close to the terminal equipment and the generated data, which can ensure that the patient's information can be delivered to the medical institution as soon as possible, and the diagnosis can be arranged.

(2) Streamlined and more reliable data

After the data is processed by fog computing technology, it will become simpler, and the requirements for transmission capacity and required bandwidth will be greatly reduced; in order to serve users in different regions, fog computing technology will be deployed on the fog nodes in each region. Service. If the service in a certain area is abnormal, the user's request will be quickly transferred to nearby nodes in other areas for service, thereby ensuring high data reliability.

(3) More nodes with wider coverage

The architecture adopted by the fog computing technology is distributed, and its remarkable feature is that no matter how weak the server node is, it will be used to play its role. It achieves better results through more nodes, which helps more "nodes" at the edge of the network to communicate and collaborate. Fog computing technology has mobility and location awareness functions, so fog nodes have a wide geographical distribution [11], can adapt to mobility applications, and support more edge nodes. These features provide convenience for the deployment of mobile services and satisfy a wider range of node access, especially $5 \mathrm{G}$ and Internet of Things applications.

(4) Organic integration of man, machine and material, high accuracy of diagnosis results

The fog server adopts classification technology based on big data mining and deep learning algorithm based on AI. Multi-source heterogeneous data processing, analysis, reasoning and presentation are all made through fog computing optimization model to make the process more intelligent, and the accuracy of case diagnosis results is higher, so as to provide doctors with efficient decision data.

\section{Conclusion}

Due to the shortcomings of the traditional cloud computing model in the application of medical big data, this paper designs a model based on fog computing optimization, that is, the intelligent front-end architecture of fog computing, to give full play to its 
advantages and characteristics, and effectively improve the data transmission, calculation and exchange. At the same time, it can avoid the data delay and huge communication overhead caused by the distance of cloud computing, making the system structure of the Internet of Things more concise, modular and intelligent. This "decentralized" distributed structured design not only ensures the accuracy and safety of data transmission, but also greatly relieves the pressure of data processing and storage on cloud servers. The model uses classification technology based on big data mining and deep learning algorithm based on AI technology to make the data calculation and analysis of the medical diagnosis system more intelligent and convenient for decision-making. At the same time, it can solve the problems in the medical system such as multi-source heterogeneous data storage and processing, improve the work efficiency of doctors, and can also alleviate the current social "difficulty in seeking medical treatment". The research results have certain value and significance.

\section{References}

[1] ZHAO Ming. Surver on Technology and Application of Edge Computing [J]. Computing Science, 2020, 47(6):268-272.

[2] LI ZHi, LIU YanZhu, etc. Research on Robustness Fog Computing Based on Complex Network [J]. Journal of North University of China (Natural Science Edition), 2017, 38(2):178-185.

[3] Fang Wei.Paradigm Change from Cloud Computing to Fog Computing [J].Journal of Nanjing University of Information Engineering (Natural Science Edition), 2016, 8(5):404-414.

[4] Xue Yuhong.Cloud Computing Drives the Development of Fog Computing. ZTE Technologies, 2017, 23(1):51-52.

[5] Yao Meiling, Wu Pengbo, etc. Discussion on Intelligent Water Meter Based on NB-IoT [J].Telecommunication Engineering Technology and Standardization, 2018, 31(6):32-35.

[6] HE Xiuli, Ren Zhiyuan, ect. Cloud and fog network for medical big data and its distributed computing scheme [J].Journal of Xi' an Jiaotong University, 2016, 50(10):71-77.

[7] Mei H, Guo Y. Toward Ubiquitous Operating Systems: A Software-Defined Perspective [J]. IEEE Computing, 2018, 51(1):50-56.

[8] SUN Ying, ZHU LiuSong. Analysis on the application of cloud computing in hospital information construction [J]. PLA Hospital Management Journal, 2014, 21(7):660-661.

[9] CHEN DongMei, LI ZHi. Fog Computing Based Hospital Information Service System [J]. Computing Science, 2015, 42(7):170-174.

[10] Li Zuozhao, Liu Jinxu . Application of Mobile Edge Computing in Internet of Vehicles [J]. modern Telecommunications Science and Technology, 2017, 47(3):37-41.

[11] WANG Yongyan, YU ZHaohu. Practice of Hospital Digital Center Construction Based on Virtualization [J]. Medical Information, 2019, 32(11):19-21. 